\title{
New force, global anisotropy and the changes in $\beta$-decay rate of radioactive elements
}

\author{
Yuriy Alexeevich Baurov ${ }^{1,2}$, Yuriy Grigoreyvich Sobolev ${ }^{3,4}$, Yuriy Vasilevich Ryabov \\ ${ }^{1}$ Closed Joint Stock Company Research Institute of Cosmic Physics, 141070, Moscow Region, Pionerskaya, 4, Korolyov, Russia \\ ${ }^{2}$ Hotwater Srl, Via Gioberti, 15, I-56024 San Miniato (PI), Italy \\ ${ }^{3}$ Joint Institute for Nuclear Researches, Moscow Region, Dubna, 141980, Russia \\ ${ }^{4}$ Nuclear Physics Institute, 25068 Rez, Prague, Czech Republic \\ ${ }^{5}$ Institute for Nuclear Research of Russian Academy of Science, 60 - the October Anniversary Prospect, 7a, Moscow 117312, Russia
}

\section{Email address:}

baurov@mail.ru (Y. A. Baurov), Sobolev@nrmail.jinr.ru (Y. G. Sobolev), Ryabov@inr.ru (Y. V. Ryabov)

\section{To cite this article:}

Yuriy Alexeevich Baurov, Yuriy Grigoreyvich Sobolev, Yuriy Vasilevich Ryabov. New Force, Global Anisotropy and the Changes in $\beta$-Decay Rate of Radioactive Elements. American Journal of Astronomy and Astrophysics. Special Issue: Global Anisotropy, Theory of Byuon, New Force, New Power System, Propulsion, Space Flights. Vol. 2, No. 6-1, 2014, pp. 8-19. doi: 10.11648/j.ajaa.s.2014020601.12

\begin{abstract}
The review of experimental studies results of a global anisotropy of physical space and new interaction in nature based on analyzing fluctuations in the intensity of the $\beta$-decay of radioactive elements are presented. The explanation of the results, based on a hypothesis as to the global anisotropy of physical space caused by the existence of cosmological vector potential $A_{G}$ in limits of theory of byuon, is given. It is shown that vector $A_{G}$ has the following coordinates in the second equatorial coordinate system: right ascension $\alpha=293^{\circ} \pm 15^{\circ}$; declination $\delta=36^{\circ} \pm 10^{\circ}$.
\end{abstract}

Keywords: Changes in $\beta$-Decay Rate of Radioactive Elements, Global Anisotropy, New Force

\section{Introduction}

There are four interactions (forces) in modern physics only: strong, weak, electromagnetic and gravitational. All modern theories are the gage models. It was shown in [1-5] and first article this Special Issue that with extraordinary variance in the action of a system of spinor and boson fields interacting with an electromagnetic field $\left(\delta \mathrm{e}_{\mathrm{o}}(x, t)\right.$ where $\mathrm{e}_{\mathrm{o}}$ - electric charge ,electric charge, $x$ - some space coordinate; $t$ - time), the potentials of physical fields that are non-measurable from the standpoint of gage theories, become unambiguous and measurable quantities within the space under study, due to violation of the gage invariance within the area of electric charge variation. One of main results of these works is that the expressions obtained for elementary particle masses appear to be proportional to the modulus of a certain vector potential. In these works, this potential is referred to as the cosmological vector potential $A_{g}$ [5], and it is assumed that if in any area the vector potential of any magnetic system has a component in the direction opposite the vector, then any matter in the area will be ejected from it. It is a new force of nature.

In classic nuclear physics the $\beta-$ and $\alpha$ - decays of radioactive elements are usually thought to be purely random processes practically not subjected to exterior influences [6, 7].
In recent years the experimental papers are appeared [8 18] in which the changes in the decay rates of various radioactive elements sufficiently above the metering errors are recorded. For example, when introducing ${ }^{7} \mathrm{Be}$ into the fullerene ${ }^{60} \mathrm{C}$ in the course of nearly 160 days, the deviation up to $0.83 \%$ in the half-life between ${ }^{7} \mathrm{Be}$ in ${ }^{60} \mathrm{C}$ and ${ }^{7} \mathrm{Be}$ in the metal was recorded [8]. In Ref. [9], a non-exponential radioactive decay of nuclei-isomers of ${ }^{125 \mathrm{~m}} \mathrm{Te}$ during uninterrupted measurements of $\gamma$-radiation in the course of a year, was also observed.

These changes are shown to have a periodic character [10 - 18]. In the paper [10], annual oscillations when investigating the half-period $\mathrm{T}_{1 / 2}$ of ${ }^{32} \mathrm{Si}$ ( $\beta$-decay) from 1982 till 1986 in the Brookhaven National Laboratory (BNL, USA), are found out. In [11] presented are results of $T_{1 / 2}$ measurements carried out in Germany, at the "PhysicalischTechnische Bundesanstalt (PTB)", with the use of an ionization chamber for ${ }^{226} \mathrm{Ra}(\alpha-$ decay).

Note that the paper [19] criticizes studies [10,11] in the context of possible influence of the thermal factor on equipment readings.

In Russia yearly [12], near-daily [12 - 18], and 27-day [12 - 18] oscillation were detected by various teams of researchers. It is to clarify that [12] does not consider 
variations of the decay products flow, but only repetitive duplication of the spectrum forms and their deviation from the Poisson distribution. Ref. [20] contains critical remarks on the work [12].

Let's explain our first works [5, 13].

For the model of weak four-fermion interaction with a current taken in the form of the sum of weak vectorial current $V^{\prime}$ and weak axial (pseudovectorial) current $A^{\prime}$ ( $V^{\prime}-A^{\prime}$ interaction), the interaction constants $C_{V}$ and $C_{A}$ have been determined in terms of the new philosophy in $[5,21]$

$$
\begin{gathered}
\left|\vec{C}_{V}\right|=\left(\frac{h c}{4 \sqrt{3} e_{0}^{2}}\right)^{2.5} \frac{(h c)^{2}}{3^{1 / 4}} \cdot \frac{1}{\left|A_{\Sigma}\right|^{2}} \\
\left|\vec{C}_{A}\right|=\frac{1}{2} e_{O}^{2}\left(\frac{h c}{4 \sqrt{3} e_{O}^{2}}\right)^{4} \cdot h c \cdot \frac{1}{\left|A_{\Sigma}\right|^{2}}
\end{gathered}
$$

Here $e_{0}, h, c$ are elementary electric charge, Planck's constant, speed of light, correspondingly.

Since the energy of $\beta$-decay electrons $E_{\beta} \sim\left|A_{\Sigma}\right|$, and the probability of $\beta$-decay $W \sim\left|C_{V, A}\right|^{2} E_{\beta}{ }^{5}$ in the framework of $V^{\prime}$ $A^{\prime}$ - interaction [22. 23], with (1) we have $W \sim\left|A_{\Sigma}\right|$.

Thus, as $\left|A_{2}\right|$ changes under the influence of vectorial potential of a magnetic system, one might expect $W$ being also changed. This result opened a way for first experiments in this direction $[5,13]$.

Later it was also shown that the changes in the $\beta$-decay rates of radioactive elements, during laboratory rotation together with the Earth, reveal in the physical space three directions fixed relative stars [15 - 18].

The present article is devoted the new force in nature and the review of experimental studies results of global anisotropy of physical space using the changing in the intensity of the $\beta$ - decay (dimension of manifestation $\approx 10^{-17}$ $\mathrm{cm})$ of radioactive elements [15-18, 24-26].

\section{The New Non-Gauge Force}

The first experimental investigations of new force were carried out at experimental facilities of the Kurchatov Institute of Atomic Energy (KIAE) and the General Physics Institute (GPI) of the Russian Academy of Sciences using high current magnets equipped with and torsion and piezoresonance balances from 1987 to 1994 [5, 17, 27-30]. The analysis these experiments discovered the new force and created a base of byuon theory [5,17]. The new force has nonlinear and nonlocal behavior, depending on the changes in a particular total potential $A_{\Sigma}\left(\left|A_{\Sigma}\right| \leq\left|A_{g}\right|\right)$, and can be represented by a particular series in terms of $\Delta \mathrm{A}_{\Sigma}[5,17,30]$.

The expression for the new force takes the form:

$$
|\vec{F}|=-2 N m_{0}\left|\vec{A}_{\mathrm{G}}\right| \cdot c^{2} \lambda(\Delta A) \cdot \frac{\partial \lambda(\Delta A)}{\partial \Delta A} \cdot \frac{\partial \Delta A}{\partial X_{1}}
$$

Here $N$ is the number of stable elementary particles in the body (electrons, protons and neutrons), $\Delta A=\Delta \mathrm{A}_{\Sigma}$.

The analysis of the specific experimental results with high field magnets (see [5, 17, 27-30]) has led to the following expression for $\lambda(\Delta A)$ :

$$
\lambda(\Delta A)=\sum_{k=1}^{\infty} \lambda_{k} \exp \left\{-\left[\frac{\Delta A}{A_{\mathrm{G}}} \cdot \frac{r}{\Delta y} \cdot\left(\frac{c t^{*}}{x_{0}}\right)^{3 / 2}\right]^{k}\right\} \cdot \Delta A^{k}
$$

Here $r$ is the radius of the circle where the test body is located on; $\Delta y$ is the difference in coordinates $y$ of the sensor and the body $[5,17,30]$;

$$
\left(x_{0} / c t^{*}\right)^{3 / 2} \text { is the part of energy } 2 m_{v_{e}} c_{0}^{2}=2 m_{0}\left|\vec{A}_{\mathrm{G}}\right| c_{0}^{2}
$$
$\approx 33 \mathrm{eV}$, which can be acted upon by the electromagnetic field potentials.

Using the linear term only in the expansion of (2) by $\Delta \mathrm{A}$, we obtain the following formula for the modulus of the new force:

$$
\mathrm{F}=2 \mathrm{Nm}_{\mathrm{ve}} \mathrm{c}_{\mathrm{o}}{ }^{2} \lambda_{l}^{2 \cdot} \Delta \mathrm{A}_{\Sigma}\left(\Delta \mathrm{A}_{\Sigma} / \Delta \mathrm{X}\right)
$$

Here $\lambda_{I} \approx 10^{-6}(\mathrm{Tm})^{-1}$ is the first coefficient of series (3).

Results from experimental investigations of the new force's characteristics using two different systems were considered in $[5,17,31]$ : one and two Sodin high precision quartz gravimeters at GAISh (Moscow State University), and a system of two quartz resonators [17]. Results from experimental studies of the luminosity of plasma created by the pulsed plasmatron in dependence on the position of its axis with respect to ambient space are presented in $[17,32$, 33]. It was shown in these experiments that the spatial distribution of the plasma emission intensity is of an explicitly anisotropic nature; i.e., a certain cone of plasmatron axis directions with an aperture on the order of $90^{\circ}-110^{\circ}$ is observed, along the generatrices of which the plasma emission reaches its maximum values. It was shown moreover that vector directed along the axis of the indicated cone had the following coordinates in the second equatorial coordinate system: right ascension $\alpha=293^{\circ} \pm 15^{\circ}$ and declination $\delta=36^{\circ} \pm 10^{\circ}$. The results from the experiments agree with earlier experiments $[5,17,27-31]$ to determine the direction of vector $\mathrm{A}_{\mathrm{g}}$ (axis of cone) and are most exact until 2011.

The cycle of investigations into the impact of the new force on the rate of $\beta$-decay of radioactive elements are performed in [5, $1318,24-26]$. But to correctly analyze the experimental results given below (It is a difference from first works, see Introduction), we immediately give a more modern interpretation of the influence of the new force on the $\beta$-decay rate (see, (4)) as a process having an inter nucleon but not inter nucleus character [34].

Scaling estimates of magnitudes of magnetic vectorial potentials of the Earth (AE) and of the Sun $(\mathrm{A} \odot)$ show them to be equal to $\sim 10^{8} \mathrm{G} \cdot \mathrm{cm}$ and $\sim 5 \cdot 10^{8} \mathrm{G} \cdot \mathrm{cm}$ in the orbit of the Earth, respectively. That is, rotation of radioactive sources around the Earth and Sun can lead to changes in the magnitude of $\mathrm{A}_{\Sigma}$ by $\sim\left|\mathrm{A}_{\mathrm{E}}+\mathrm{A} \odot\right| /\left|\mathrm{A}_{\Sigma}\right| \approx 10^{-3},\left(\left|\mathrm{~A}_{\Sigma}\right| \approx \mathrm{A}_{\mathrm{g}} \approx\right.$ $\left.1,9510^{11} \mathrm{Gs} \mathrm{cm}\right)$.

Since the first approximation of the new force (4) includes not only $\Delta \mathrm{A} \Sigma$ but $\frac{\partial \Delta A_{\Sigma}}{\partial x}$, too, the change of the probability of 
$\beta$-decay $\Delta \mathrm{W}$ may be assumed to be proportional $\Delta A_{\Sigma} \frac{\partial \Delta A_{\Sigma}}{\partial x}[15$, 17] but not only to $\Delta \mathrm{A} \Sigma$ as was previously considered in Introduction. Thus it is assumed that the new force can act on the decay of neutron. Let us explain this.

As is known [34], the neutron has a magnetic moment $\mathrm{Mn} \approx 10^{23} \mathrm{erg} / \mathrm{G}$. The scaling estimations show that in connection with such its magnetic moment, an enormous value $\frac{\partial \Delta A_{\Sigma}}{\partial x} \approx 10^{16} \mathrm{G}$ may be expected in the vicinity of the neutron, but at that the value of $\Delta \mathrm{A}_{\Sigma}$ from the magnetic field of neutron is not large and equals $10^{3} \mathrm{G} \cdot \mathrm{cm}$. Since during rotation of radioactive sources together with the Earth around its axis and the motion of the Earth around the Sun the value of $\Delta \mathrm{A}_{\Sigma}$ can vary over five orders at the place of these sources from the action of potentials $A_{E}$ and $A_{\odot}$ onto $A_{\Sigma}$, the combined influence of two factors from the Earth and the Sun as well as of magnetic field of neutron can create such new force which will be able to act on the neutron life time.

\section{New Force and the Changes in $\beta$ - Decay Rate of Radioactive Elements, First Experiments}

In connection with rich experimental material on the subject we will only briefly show results of initial investigations. In Ref. [5, 13, 17], with the use of scintillation procedure, near daily periodic variations of the $\beta$-decay rate of ${ }^{60} \mathrm{Co},{ }^{137} \mathrm{Cs}$ were first detected. In Figs. 1, 2, time variations of decay numbers of ${ }^{137} \mathrm{Cs}$ and ${ }^{60} \mathrm{Co}$ in the runs of experiments of (19-23).04.1994 and (22-23).04.1994, are shown (from an independent measuring channel).

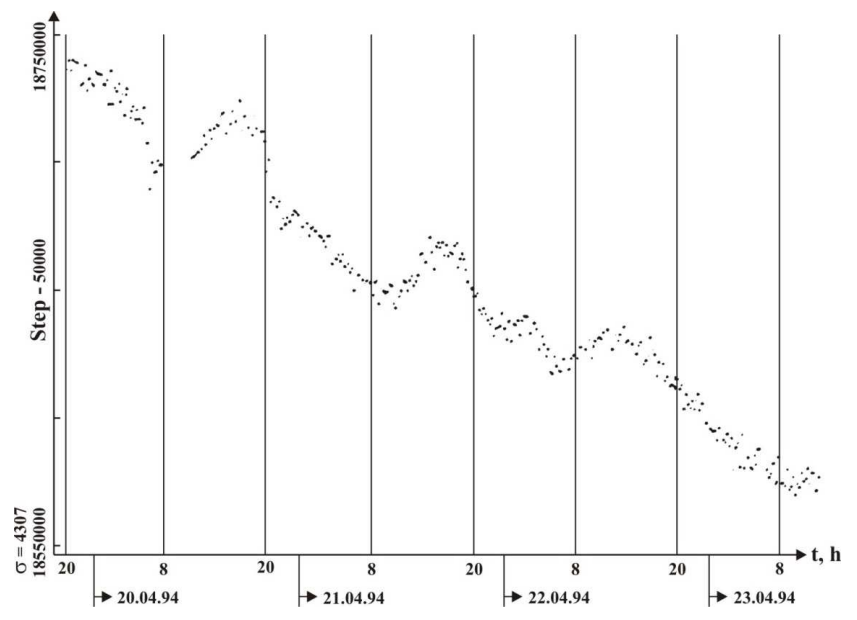

Fig. 1. Time variation of Cs-137 decay number in the run of measurements on Apr.19-23,1994.

As is seen from Fig. 1, a following effect was observed: in the morning hours in parallel with the decrease of readings of an analyzer, a symmetric growth of them was registered. The magnitude of oscillations was equal to $\sim 25$ thousand photons (at an initial level of $\sim 18.6$ millions in $\sim 16$ min) which corresponds to $\sim 6 \sigma$ ( $\sigma$ is the standard deviation, $\sigma=4307$ ).
The repetition of the effect in the course of the total experimental cycle points to the fact that it is not a random one.

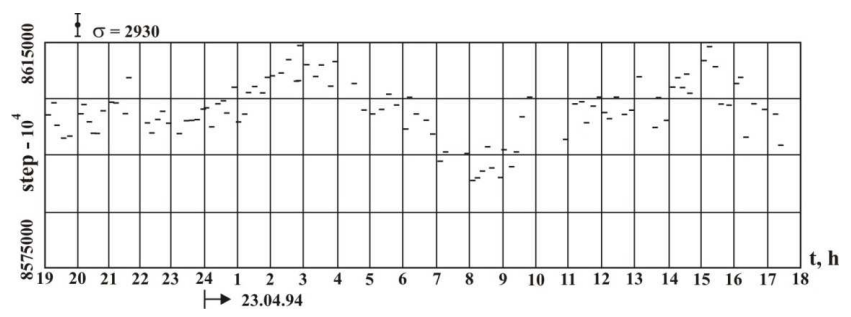

Fig. 2. Time variation of Co-60 decay number on Apr.22-23,1994.

For ${ }^{60} \mathrm{Co}$ (Fig.2), similar oscillations had an amplitude of 20 thousand events at an average level of $8.6 \cdot 10^{6}$ (at the exposition time of $\sim 7 \mathrm{~min}$ ), i.e. approximately $7 \sigma$.

The tangent lines to the Earth's parallel of latitude (along the parallels, the vector $A_{E}$ is directed) at the place of observation of minimum intensity of the $\beta$-decay and of accompanying $\gamma$-quanta at 8-10 hours of Moscow time gave, the direction of the new force with an angle $\alpha \approx 240^{\circ}-270^{\circ}$.

In August-September, 1996, and in February, 1997, two runs of experiments on investigation of ${ }^{90} \mathrm{Sr} \beta$-decay count rate were carried out at in the Laboratory for nuclear reactions (FLNR) named after G.N.Flerov, at the Joint Institute for Nuclear Researches (JINR, Russia, Dubna town) $[5,17,35]$. The measurements were made by the scintillation procedure with a ${ }^{90} \mathrm{Sr}$-source. With the aim of protection from an external magnetic field, the scintillation detector and preamplifier were positioned in a permalloy envelope at a distance of $3 \mathrm{~cm}$ from the collimated source. The entire set was situated in a thermostable room where fluctuations in temperature were no more than $2^{\circ} \mathrm{C}$ for the whole time of measurements. The temperature conditions were checked no more rarely than two times daily. Before each session of measurements, the installation was forced into an operating mode during a week.

The aim of the experiment was to detect the daily periodicity and the degree of unevenness of decay number distribution in astronomical time. The presence of periodical components was revealed by two methods. First, the standard Fourier analysis of the series was carried out. Second, the following procedure was used to refine the distribution of $\beta$ decay numbers over the astronomical day. Each moment (minute) of measurement was represented as a point of a circle (corresponding to an astronomical day) and expressed in degrees, so that the whole series could be "coiled" around that circle. Thus, each measurement was related to a certain time of day (in degrees). If the quantity to be measured is isotropic in time, then the distribution thereof over the circle will be uniform, and the hypothesis for uniformity of said distribution may be verified by statistical methods. For this purpose we used the Kolmogorov-Smirnov's test based on computation of maximum difference in the theoretical and experimental distribution functions and on comparison of this difference with a tabulated value. Should this difference be sufficiently large for a given sample size and an accepted 
significance level, the conclusion may be drawn that the experimental and theoretical distributions are different. In our case with the theoretical distribution chosen being uniform, this signifies the non-uniform distribution of the metered quantity in time. The Kolmogorov-Smirnov's test is convenient also in view of obtaining, in parallel with estimation of confidence level, a point (day time) at which the deviation from uniformity is the maximum. The graphically results are shown in [5, 17, 35]. The Fourier spectrum was shown a half-week and 24 -hour periods. The Kolmogorov-Smirnov's test defined that the maximum of said events corresponds to an angle about $280^{\circ}$ degrees.

Let us describe an uninterrupted experiment on investigating variations in the rate of $\beta$-decay of ${ }^{137} \mathrm{Cs}$ and ${ }^{60} \mathrm{Co}$, which was carried out at JINR (Dubna) from 9.12.1998 till 30.04.1999 [16, 17].

The main shortage of experiments was that when analyzing the final result it was impossible exactly to understand which part of that result was due to the "internal life" of the installation itself, and which might be caused just by the phenomenon of interest. In addition the duration of those experiments was no more that two weeks which did not allow to analyze long-period harmonics.

The aim of the experiment in consideration was to tackle the above questions.

The schematic of the installation was shown in [16, 17].The installation consisted of three scintillation detectors two of which were standard spectrometric scintillation detecting units BDEG 2-23 on the basis of $\mathrm{NaI}$ (Te)scintillator $(63 \mathrm{~mm}$ in diameter, $63 \mathrm{~mm}$ in height) and a photomultiplier FEU-82 with standard divider. One of those units was used for detecting the background radiation, another measured the $\gamma$-radiation from the ${ }^{137} \mathrm{Cs}$ source. The third detector was a BGO-scintillator $(46 \mathrm{~mm}$ in diameter, $60 \mathrm{~mm}$ in height) with a photomultiplier FEU-143 and standard divider, this one was intended for registration of ${ }^{60} \mathrm{Co} \gamma$-radiation.

To attenuate the influence of magnetic fields on the photomultipliers, the detectors were shielded. The shields were cylinders (of inner diameter $10 \mathrm{~cm}$, height $40 \mathrm{~cm}$ ) made from ten layers of annealed sheet permalloy $0.5 \mathrm{~mm}$ thick.

The detectors were arranged in such a manner that the FEU photocathodes were from them at a distance of one half the height of the cylinder. The $\gamma$-sources were placed immediately on the face surface of scintillators through the center of entrance window.

All detectors, including a temperature-sensitive element, were positioned inside a metallic cube $\left(40 \times 40 \times 50 \mathrm{~cm}^{3}\right)$ curved as an additional magnetic shielding. The thickness of steel walls of the cube was $3 \mathrm{~mm}$. The detectors with $\gamma$ sources were in circled with lead protection $5 \mathrm{~cm}$ thick.

The system of data registration consisted of two subsystems gathering information in the course of experiment. The first subsystem accumulated count rate information in ten-second time intervals from six paths of scintillation detectors as well as information on temperature, voltages of power source (high voltage for photomultipliers, $\sim 6 \mathrm{~V}$ in the KAMAK-crate, and $\sim 24 \mathrm{~V}$ ), and impulse noises in the crate's power supply circuits. The second subsystem of data accumulation was intended to detect "labeled" energy distributions from scintillation detectors for testing stability of their amplitude distribution parameters (thresholds of discriminators, amplitude distribution forms, etc.).

On the basis of long-termed investigations performed with the use of scintillation $\gamma$-detectors, some changes in the $\beta$ decay rate of radioactive elements with periods of 24 hours and 27 days, were detected. The use of scintillation procedures methods gives results with much better than $5 \%$ level of significance using the Kolmogorov-Smirnov's test. The deviation in the changes of $\beta$ - decay rate of radioactive elements separate the anisotropic direction in the physical space during the rotation of a laboratorial system together with the Earth with $\alpha$ about $280^{\circ}$ degrees.

It is important to note that, for the time period being considered (from December 9, 1998, to April 30,1999), the anisotropy effect in question (directions of the above tangent lines determined with respect to stars and associated with the appearance of extremes in changes in the rate of the beta decay of radioactive elements with a period of about $24 \mathrm{~h}$ ) became weaker and virtually disappeared from the middle of January 1999.

\section{Experiments on the Investigation of Changes in the in $\beta$-Decay Rate of Radioactive Elements with the Use of Germanium-Lithium Detectors}

The results of experimental investigations of the $\beta$-decay of ${ }^{137} \mathrm{Cs}$ and ${ }^{60} \mathrm{Co}$ with the aid of detectors allowing to minimize the statistical and systematical uncertainties and more clearly reveal the physical phenomenon, are presented in the $[15,17]$.

Measurement procedure.

The aim of the experiment was to measure, for greater plausibility of its results, changes in the $\beta$-decay rate simultaneously at different points on the Earth and uninterruptedly during a long period of time.

The town Dubna (Russia)and Troitsk (Russia) are nearly at the same (Moscow's) meridian, the distance between them is $\sim 140 \mathrm{~km}$. The experiment lasted from the middle of February till the middle of May, 2000.

The experimental technique (schematic diagram, electronic equipment, measurement of backgrounds) at Dubna was the same as in the experiment described in detail in [54], and differed from the latter only in that a $\mathrm{Ge}(\mathrm{Li})$ detector was used. As before (when using a scintillation detector), the intensities of $\gamma$-transition from an excited level of a daughter nucleus with energy of $0.661 \mathrm{MeV}$ in the course of the $\beta$-decay of ${ }^{137} \mathrm{Cs}$, was measured. The value of the integral of $\gamma$-quanta counts entered into the memory of a computer every $10 \mathrm{~s}$.

As distinguished from the technique of long-term measurements of $\beta$-decay rate with an scintillation detectors 
$[5,13,14,16,17]$, the $\gamma$ - registration following the $\beta$-decay of the investigated radioactive nuclei with a $\mathrm{Ge}(\mathrm{Li})$-detectors made it possible to substantially improve the stability and reliability of long-term measurements. At Troitsk, a Ge(Li)detector with a volume of $100 \mathrm{~cm}^{3}$ was used for measuring the $\gamma$-spectra with energies of $1.117 \mathrm{MeV}$ and $1.332 \mathrm{MeV}$ accompanying the $\beta$-decay of ${ }^{60} \mathrm{Co}$. A radioactive source was placed beyond of the vacuum volume at a distance of $7 \mathrm{~mm}$ from the sensitive surface of the detector. To protect the detector and preamplifier from the possible influence of alternating high-frequency and magnetic fields, they were closed by covers from permalloy and electrolytic copper, and a $10 \mathrm{~cm}$ layer of lead served as a shielding from the natural radioactive background. The signal time constant of the input signal was equal to $0.5 \mu \mathrm{s}$ at a gain factor of $10-20$ which led the influence of amplitude overload of electronic paths to a minimum.

To record the amplitude spectra, a fast analog to digital converter (ADC) with off-line storage built into a personal computer (PC), was used. A control program gave the time of measuring each spectrum (600s), start time, storage instruction, noted the time of transcription of a next spectrum to the PC memory, zeroed the off-line storage, and started a new measurement. The program worked in a cycle so that the information sequentially accumulated in the memory of PC through a long time. The final processing of information was made off-line by integration over the spectrum in various intervals of energy from the first channel to the maximum energy of photopeak (or only the peak itself) with the resulting formation of a sequence of numbers reflecting the time dependence of the $\beta$-decay rate. The statistic-average digital load of the detector was no more than $(2 \div 3) \cdot 10^{4}$ counts per second, i.e. corresponded to the optimum working conditions of the instrumentation. The statistical accuracy obtained at a one point was $0.03 \%$ for the radioactive decay of ${ }^{60} \mathrm{Co}$.

Thus the measurements were made simultaneously by two identical $\mathrm{Ge}(\mathrm{Li})$-detectors with two independent and different systems of information storage in natural conditions spaced $140 \mathrm{~km}$ apart. One detector measured the decay of ${ }^{137} \mathrm{Cs}$, the other did that of ${ }^{60} \mathrm{Co}$.

Results and discussion.

In Fig. 3 and 4 the results of simultaneous measurement of the $\gamma$-counts rate of at Dubna $\left({ }^{137} \mathrm{Cs}\right)$ and at Troitsk $\left({ }^{60} \mathrm{Co}\right)$, respectively, at a period from $16^{24}$ (Moscow time) of 15.03.2000 till 10.04.2000 inclusive.

To correlate the results of two experiments, the values of flows from Dubna were averaged over 600s time intervals, like those at Troitsk, and additionally low-frequency filtration was made. The results from Troitsk did not processed altogether. As is seen from Fig.4, the change in the $\gamma$-count rate ranged in that experiment up to $\sim 0.7 \%$ of the statistical average. At Dubna these changes were no more than $0.2 \%$. It should be noted that the flow jump in the vicinity of $2180 \mathrm{~min}$ (Fig.3) was caused by a technological

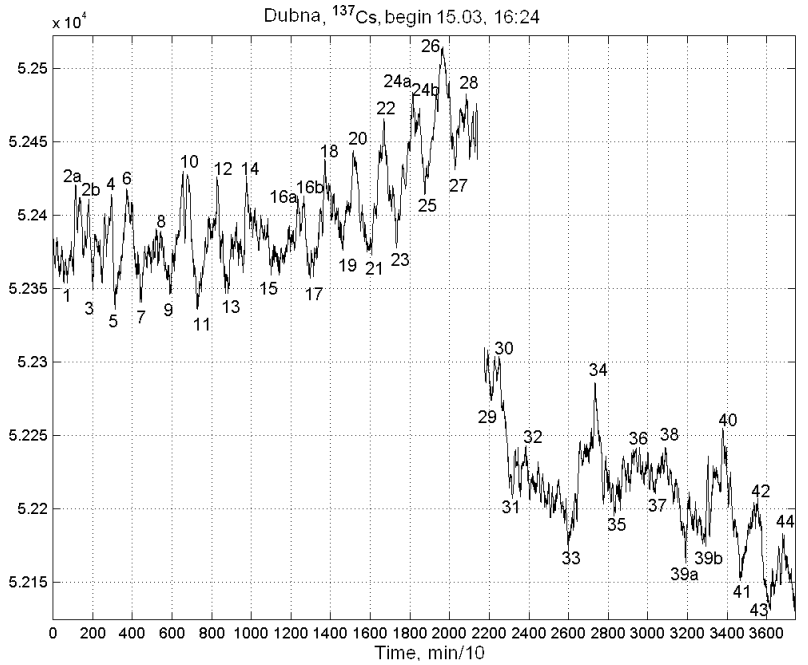

Fig 3. The variation of the flow of $\gamma$-quanta accompanying the $\beta$-decay of ${ }^{137} \mathrm{Cs}$, with the time (JINR, Dubna).
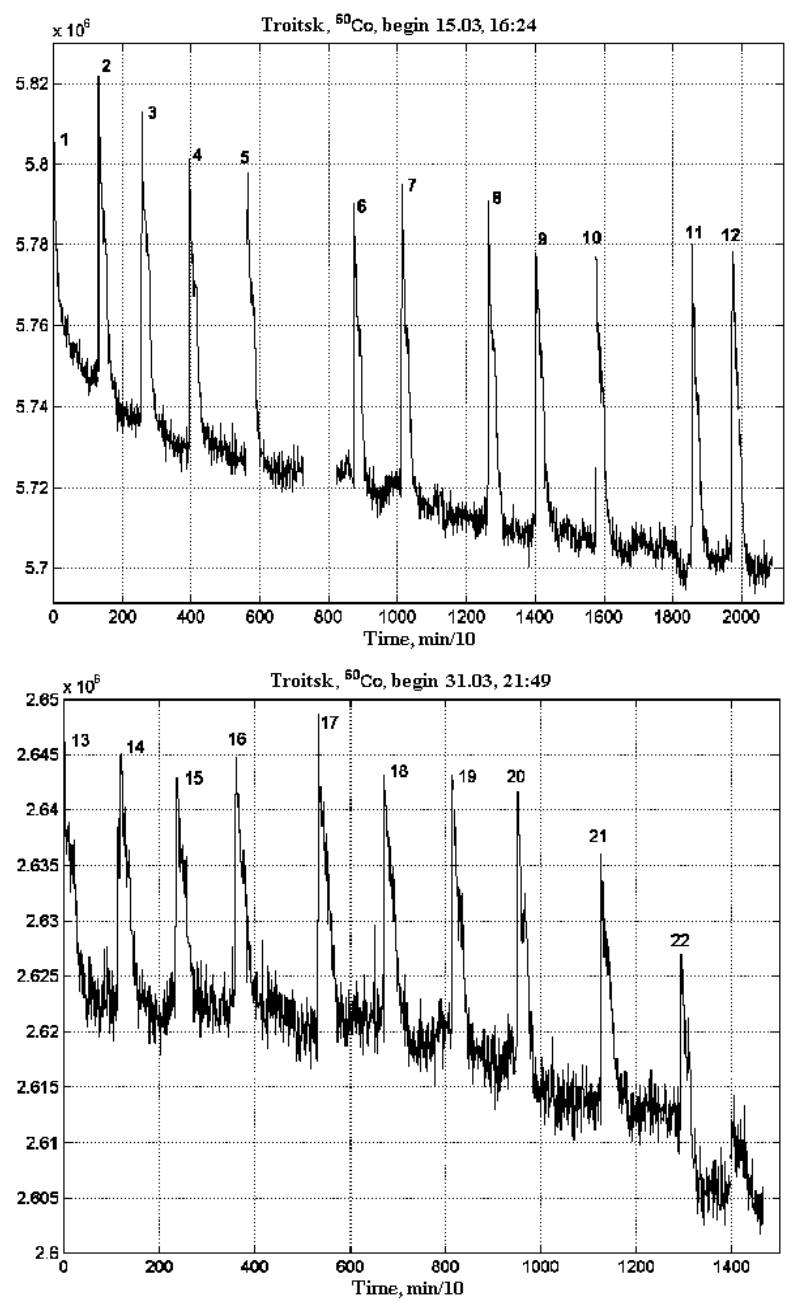

Fig. 4. The variation of the flow of $\gamma$-quanta accompanying the $\beta$-decay of ${ }^{60} \mathrm{Co}$, with the time (INR, Troitsk).

change of the radioactive source relative to the $\mathrm{Ge}(\mathrm{Li})$ detector in the process of refilling a Dewar flask with liquid nitrogen. The difference in the values of $\gamma$-count rate changes in the experiments at Dubna and Troitsk is probably 
connected with dissimilar measuring procedures: in the former case the $\gamma$-quanta were counted at a fixed energy threshold but at Troitsk one measured the total amplitude spectra and then determined the total $\gamma$-counts by integration over the spectrum. The difference of deviations can be explained also by that magnetic moments of the nuclei of ${ }^{60} \mathrm{Co}$ and ${ }^{137} \mathrm{Cs}$. However that difference can be possibly associated with the different latitude position of the experimental setups.

The observed regular structure in the time dependence of the $\beta$-decay rate for nuclei ${ }^{137} \mathrm{Cs}$ and ${ }^{60} \mathrm{Co}$ can be, in general, explained by the following reasons:

a) temporal instabilities of the electronic recording paths;

b) outside influences and those connected with the human activity;

c) unknown physical processes in the $\mathrm{Ge}(\mathrm{Li})$-detectors themselves in the course of long-term measurements;

d) a "cosmological" factor acting on the process of the $\beta$ decay of nuclei.

Consider each reason separately for the setup of Troitsk (that of Dubna, as was mentioned above, is considered in detail in $[16,17])$.

a) The structure of changes in the count rate is such that it cannot be explained by the slow variations of the supply-line voltage $(220 \mathrm{~V})$. Besides, the low-voltage supply of the electronic circuits was stabilized with an accuracy of $3 \%$ and did not vary when the supply-line voltage was changed within $15 \%$. As is known, the spectrometric characteristics of $\mathrm{Ge}(\mathrm{Li})$-detectors practically do not depend on insignificant variations of high-voltage supply.

b) As for the structural changes in the $\beta$-decay count rate due to variations of some external influences, daily variations of intensity of cosmic radiation, changes in the room temperature, etc, we can say that the detectors themselves as well as the channels of electronic paths were carefully protected against variable, alternating, and leakage electromagnetic and high-frequency fields. The natural background of the $\mathrm{Ge}(\mathrm{Li})$-detector was as little as about $0.1 \%$ of the $\beta$-decay count rate of the radioactive sources investigated. That is, only a periodical 7-8 times increase of the background could explain the structure observed in the $\beta$-decay count rate. But the count rate of the background was constant in the limits of the statistical accuracy. To evaluate experimentally a possible influence of count overloads (idle time) of the electronic paths when measuring the background, one simultaneously fed to the input of the preamplifier a signal from a generator with an amplitude equal to that of the photopeak but with frequency 50-100 times more than under the operating conditions. For such spectra, there were no peculiarities in the time dependence of the count rate.

c) It is possible that in the material itself of the Ge(Li)detector some yet unknown physical processes take place during the long-term exposure to radiation which lead to accumulation of charge in "internal" capacities, then to a break-down and relaxation of charge. This would correspond to the observable structure form in the time dependence if the "time constant" were close to 24 hours. In such a case the amplitude spectrum of $\gamma$ quanta would be perturbed, too, in those intervals of time but this was not observed in the experiments. As to an influence of capacitive coupling in the amplifying section itself, control measurements were carried out with the use of non-capacitive current amplifiers, and a similar structure with the same value was found in the time dependencies obtained.

Finally consider a possible influence of the cosmological factor on nuclear processes on Earth. As was said [5, 13, $14,17]$, it can be associated with a new suggested interaction of objects in nature caused by the existence of the cosmological vectorial potential $\mathrm{A}_{\mathrm{g}}$.

In Fig. 5 and Fig. 6, the small circles denote places of observation of maximum flows of $\gamma$-quanta at the $\beta$-decay of ${ }^{60} \mathrm{Co}$ at Troitsk as well as the maximum and minimum flows for the decay of ${ }^{137} \mathrm{Cs}$ at Dubna, respectively.

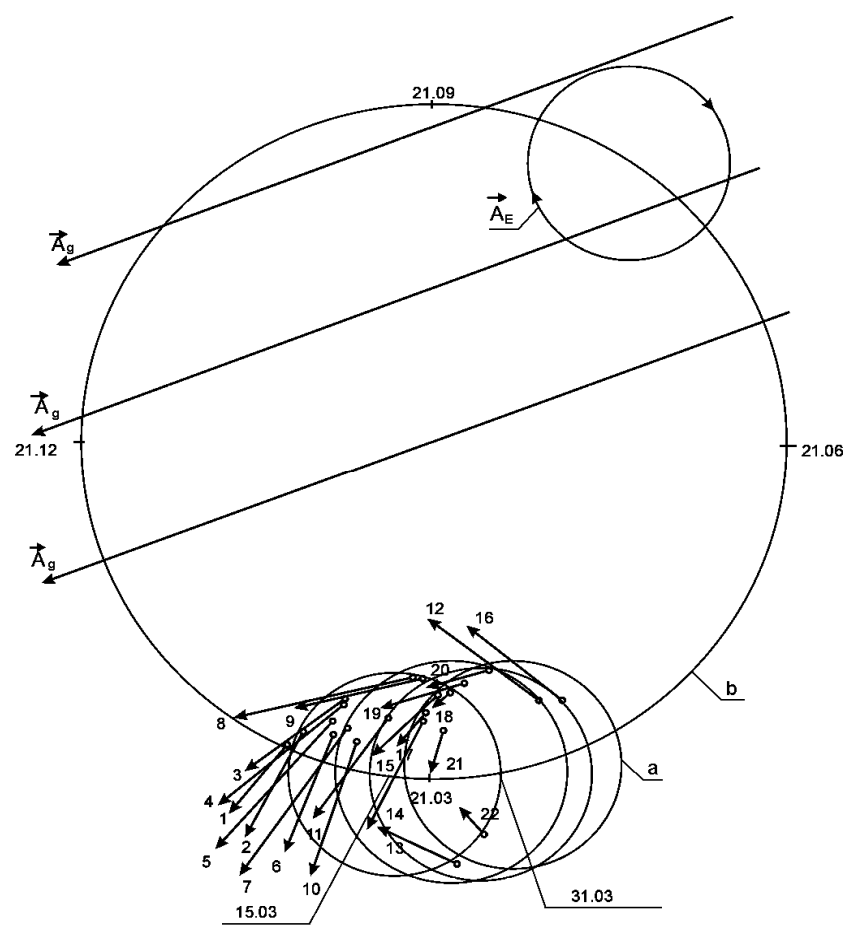

Fig. 5. The spatial positions of sites where the clearly expressed extrema in the magnitude of the flow of $\gamma$-quanta in the experiment with the $\beta$-decay of ${ }^{60}$ Co, were observed (see Fig. 4).

$\leftarrow \bullet$ - the site of the maximum flow of $\gamma$-quanta with the indication of the direction of action of the new force drawn along the tangent line to the parallel of latitude;

a - the trajectory of motion of the radioactive source rotating together with the Earth

b - the trajectory of motion of the Earth and the radioactive source around the Sun;

21.03 etc. -the point of the vernal equinox and other characteristic points of the trajectory "b";

$A_{E}$-the direction of the vectorial potential of the magnetic field of the Earth's dipole;

$\mathrm{A}_{\mathrm{g}}$ - the direction of the cosmological vectorial potential. 


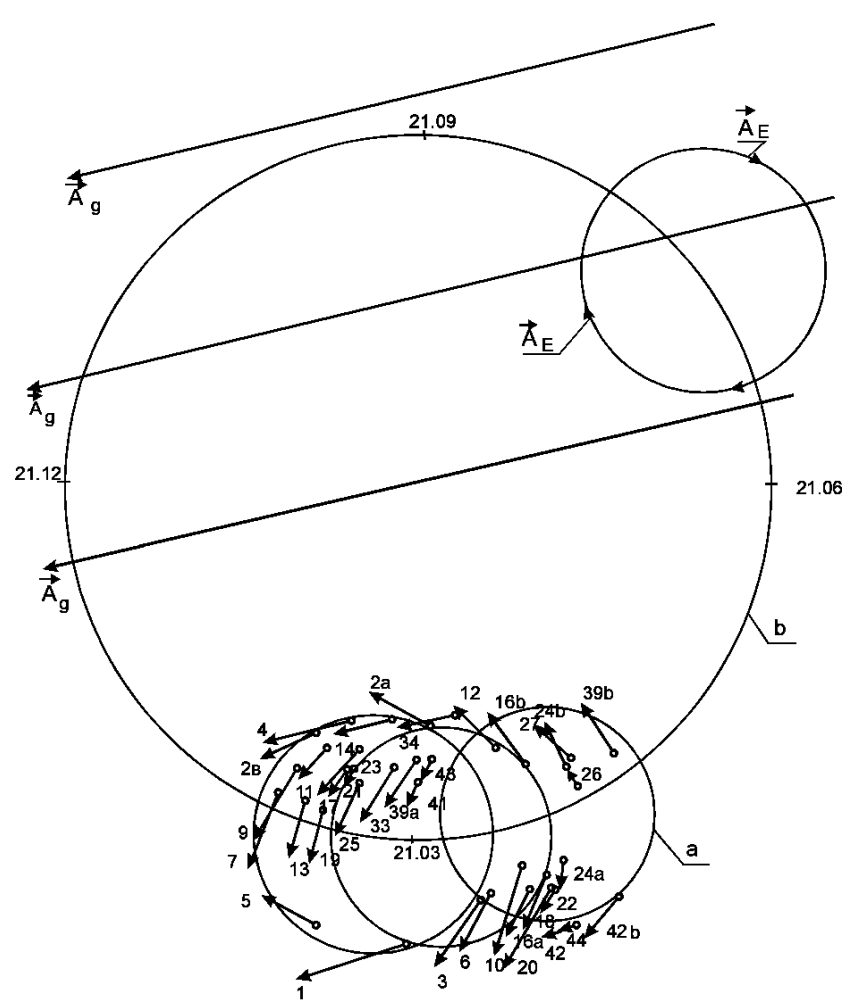

Fig. 6. The same as in Fig.5 but for minimum and maximum flows of $\gamma$ quanta during the decay of ${ }^{137} \mathrm{Cs}$ ( see Fig.3).

The numbered maxima and minima of flows of $\gamma$-quanta in Fig. 3 and Fig. 4 correspond to number of arrows in Figs.5, 6 drawn from place of observation of an extremum tangentially to the parallels of latitude (along which the vectorial potential of the magnetic field of the Earth's dipole is directed)

As is seen from Fig. 5 and Fig. 6 the total set of numbered arrows can be clearly divided, by their directions, into three subsets with an accuracy of $\pm 15^{\circ}$.

In Fig. 6 we have the subsets $D_{1}(7,9,11,13,17,19,21$, $23,25,33,39 \mathrm{a}, 41,43,3,6,10,16 \mathrm{a}, 18,20,22,24 \mathrm{a}, 42), \mathrm{D}_{2}$ (2b, 4, 14, 34, 1, 42, 44), $\mathrm{D}_{3}(2 \mathrm{a}, 12,16 \mathrm{~b}, 24 \mathrm{~b}, 39 \mathrm{~b}, 5)$. In Fig. 5 these are: $\mathrm{T}_{1}(1,2,3,4,5,6,7,10,11,15,17,18,21)$, $T_{2}(8,9,19,20), T_{3}(13,22,12,16)$. The subset $D_{1}$ is seen to be in correspondence with $T_{1}, D_{2}$ is with $T_{2}$, and $D_{3}$ is with $\mathrm{T}_{3}$. Thus we see, in the same time interval and at the different experimental setups being $140 \mathrm{~km}$ apart, a similar pattern of behavior of the maxima and minima of the $\beta$-decay rate for the different radioactive elements ${ }^{137} \mathrm{Cs}$ and ${ }^{60} \mathrm{Co}$.

The emergence of the set $D_{2}, T_{2}$ is easily explicable on the basis of a hypothesis of equiprobable distribution of directions of neutron magnetic moments in the nuclei and total substance of radioactive source. That is, in any source always there are neutrons with the magnetic moments being perpendicular to the vector $A_{g}$ and hence the lines of the vectorial potential of magnetic field of the neutron will be always directed, in some region of space, under an efficient angle of $\sim 140^{\circ}$ to $\mathrm{A}_{\mathrm{g}}$ corresponding to the maximum value of $\frac{\partial \Delta A_{\Sigma}}{\partial x}$ and hence of the new force. At that the direction of the subset of arrows $D_{2} \quad T_{2}$ gives that of the vector $A_{g}$ with $\alpha \approx 285^{\circ} \pm 15^{\circ}$. The subsets of arrows $\mathrm{D}_{1} \mathrm{~T}_{1}$ and $\mathrm{D}_{3} \mathrm{~T}_{3}$ point to directions of the cone generatrices of new force action.

Thus the experiment considered has qualitatively confirmed the results of earlier experiments on investigation of changes in the $\beta$-decay rate of radioactive elements as well as has refined the direction of the vector $A_{g}$.

Thus, the experiments reported here, which were performed at different research centers by different groups of experimentalists who used different experimental procedures, indicate that a long-term observation of the beta decay of radioactive elements reliably reveals changes in the betadecay rate with a period frequently equal to $24 \mathrm{~h}$ and $27 \mathrm{~d}$. However, these periods always arise when, at the instant of observation of the effect, the lines tangent to the parallels on the Earth's surface at the position of the respective laboratory pass through three specific spatial directions (see Fig. 5,6). The time interval within which extreme changes in the betadecay rate are observed may be $18.5 \mathrm{~h}$ and about $30 \mathrm{~h}$, in addition to the aforementioned $24 \mathrm{~h}$.

\section{New Force and the Last Results of Experimental Investigation of ${ }^{60} \mathrm{Co}$ $\beta$-Decay Rate Variation}

This experiment was carried out in JINR, Dubna, Russia from 28.12.2010 till 08.02.2012 [25]. Ref. [24] presents preliminary results of the experimental study of the ${ }^{60} \mathrm{Co} \beta$ decay rate in period 2010 February-August which confirmed the presence the two of the above three spatial directions [15, 17].

In 2010 the experimental installation was being debugged, during which in particular the influence of the temperature on the results was investigated. Analysis of the [24] paper results has shown the necessity of placement the experimental installation into the thermostatic regulator with high level stability of the temperature, while using scintillation detectors.

The present paper is a continuation of the investigations set forth in [5, 13-18] and [24], and designed to develop the experimental investigation method without considerable expenses to minimize systematic errors of the experiment in registration of ${ }^{60} \mathrm{Co} \beta$-decay rate changes within a day or longer intervals.

The experimental installation and choice of $\beta$ source

The choice of radioactive source was determined by the results of previous experiments and the assumption of a possibility to maximize the action of the assumed new natural force responsible for changes in the $\beta$-decay rate of radioactive elements and to minimize systematic errors. The analysis of experimental results of the works [5, 13-18] has shown that the $\beta$-decay rate deviation value is largest for the radioactive elements having the nucleus with the maximum magnetic moment. This condition corresponds with the action maximum of the new force. Thereupon the radioactive $\beta$ source ${ }^{60} \mathrm{Co}$ was chosen for the investigation. The presence of two lines in its spectrum (1.117 MeV and 1.332 MeV) 
allows synchronously observe the above indicated $\gamma$-quanta accompanying the $\beta$-decay of ${ }^{60} \mathrm{Co}$ and minimize electronicsdependent systematic experimental errors.

Let's shortly describe experimental installation.

The experimental installation is placed in the thermostatic regulator that keeps temperature within the limits $30.6^{\circ} \mathrm{C} \pm$ $0.1^{\circ} \mathrm{C}$. The detecting module of installation contains a radioactive $\beta$ source ${ }^{60} \mathrm{Co}$ and is two-arm scintillation $\gamma$-spectrometer in hermetic case. Either of the two detectors records about 32 thousand $\gamma$-quanta per second. $\operatorname{LaBr}_{3}(\mathrm{Ce})$ crystal is used as scintillation counter, relative energy resolution of which equals $3 \%$. The crystal has $25 \mathrm{~mm}$ in diameter and $12 \mathrm{~mm}$ in height. The flowchart of the $\gamma$-spectrometer electron system is shown in Fig. 7. The electron system consists of two identical microprocessorbased information processing assemblages and common control-feed assembly on the motherboard. To receive synchronization signal, the apparatus has an interface CAN on the motherboard.

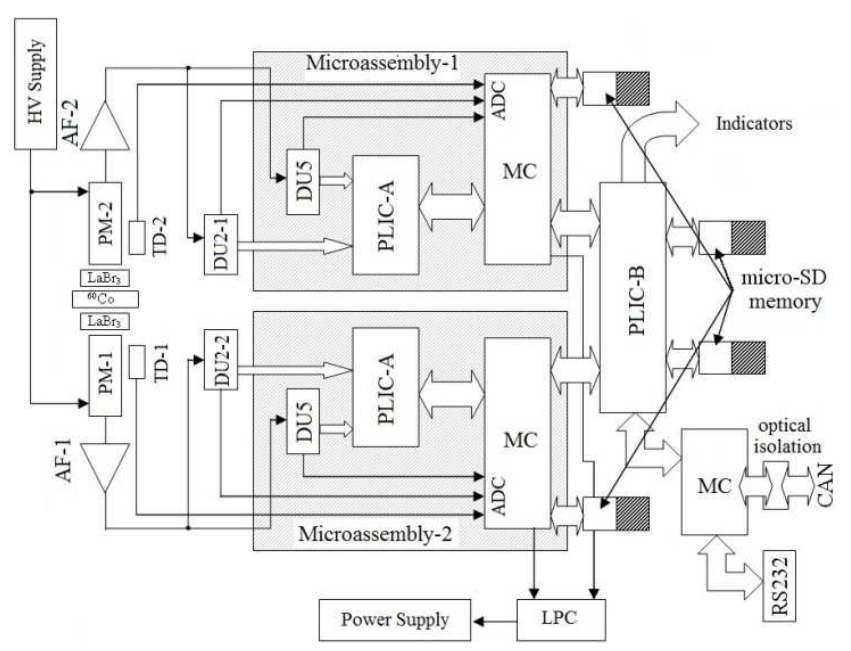

Fig. 7. Gamma-spectrometer flowchart.

HV - High Voltage, PM - photomultiplier, AF - amplifiesforms, TD - thermal detector, DU - discriminator unit, PLIC - programmable logic integrated circuit, ADC - analogdigital convertor, $\mathrm{MC}$ - microcontroller, LPC - power supply latching circuit.

The light pulses generated in the active zone of scintillation detectors are transformed into electron pulses by multiplier photocells PM-1 and PM-2. The signals from MPs are additionally amplified and formed by amplifies-forms AF-1 and AF-2. The formed pulses are further transmitted to the discriminator units DU2-1, DU2-2, and two microprocessor assemblies containing a microcontroller MC, discriminator units DU5, and a programmable logic integrated circuit (PLIC). Each discriminator unit DU2-1, DU2-2 contains two discriminators which thresholds are adjusted for detection of the pulse in the range from $0.3 \mathrm{MeV}$ to $3 \mathrm{MeV}$. The coincident pulses in both channels are recorded by counters. The reference voltages of the discriminators come to the microcontroller ADC and get stored there. The discriminator unit DU5 contains five discriminators adjusted for pulses registration in the energy ranges $>0.3 \mathrm{MeV},>1.1 \mathrm{MeV},>1.3 \mathrm{MeV},>1.6 \mathrm{MeV}$, and 2.8 $\mathrm{MeV}$ for further spectral analysis. The reference voltages of this discriminator are transmitted to the microcontroller ADC.

The pulses count is summarized each 10 seconds interval, then read out by microcontroller and recorded into the external memory realized on microdrives that allow data recording onto replaceable 2 GB memory cards. The temperature is also registered at several specially separated points of the apparatus and recorded. Information is read out from the memory cards within the interval from 10 days to 3 months.

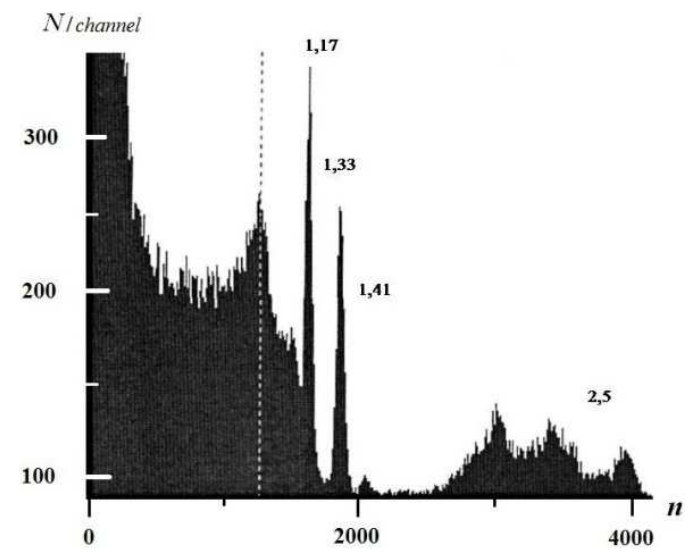

Fig. 8. ${ }^{60} \mathrm{Co}$-ray radiation spectrum, recorded by use of $\mathrm{LaBr}_{3}(\mathrm{Ce})$ scintillation counter.

The number of $\gamma$-quanta per channel (N/channel) is plotted against the axis of ordinates, and the number of $n$-th channel - against the axis of abscissas. Characteristic lines ${ }^{60} \mathrm{Co}(1.17 \mathrm{MeV}$ and $1.33 \mathrm{MeV})$, natural isotope impurity ${ }^{40} \mathrm{~K}$ $(1.41 \mathrm{MeV})$ and impurity of heavy radioactive elements $(2.5 \mathrm{MeV})$ are indicated with numbers.

Characteristic $\gamma$-ray radiation spectrum is shown in Fig. 8. It should be noted that the spectrometer energy calibration can be easily done. Three lines $1.173 \mathrm{MeV}, 1.332 \mathrm{MeV}$ and $1.41 \mathrm{MeV}$ are clearly separated in the spectrum. The latter belongs to ${ }^{40} \mathrm{~K}$ natural isotope impurity. Around the energy range $\approx 2.5 \mathrm{MeV}$ there is an enhancement caused by overlapping of the signal from natural impurity of heavy radioactive elements. These signals does not interfere normal running of the experiment, moreover it can be used in spectrometer calibration.

\section{The results of experiment}

The present paper contains results of the experiment on the study of deviations in ${ }^{60} \mathrm{Co} \beta$-decay from the standard exponent. The exposition covers the period from December 28, 2010 to February 08, 2012.

Fig. 9 shows average daily results for $\gamma$-quanta flows, registered by the first channel with the threshold $0.3 \mathrm{MeV}$, as well as temperature values in relative units. The second channel gives the similar results. Discrete steps in diagram (1) and temperature (2) variations in the first half of the picture correspond to replacement of the installation memory cards. Abrupt change of the temperature (at a level of $2.7 \%$ ) at the 
end of July was caused by the thermostatic regulator failure to maintain temperature due to heat wave in Moscow that time, in consequence of which the temperature of premise exceeded that preset for thermostatic regulator $\left(30.6^{\circ} \mathrm{C} \pm\right.$ $0.1^{\circ} \mathrm{C}$ ). Temperature variations level (except for the above abnormality and short periods of memory cards removal) didn't exceed $0.2^{\circ}$. The data analysis reveals correlation between the intensity of $\gamma$-quanta and the temperature value. The corresponding small correction is applied to the quanta counting value and does not influence obtaining the consistent results.

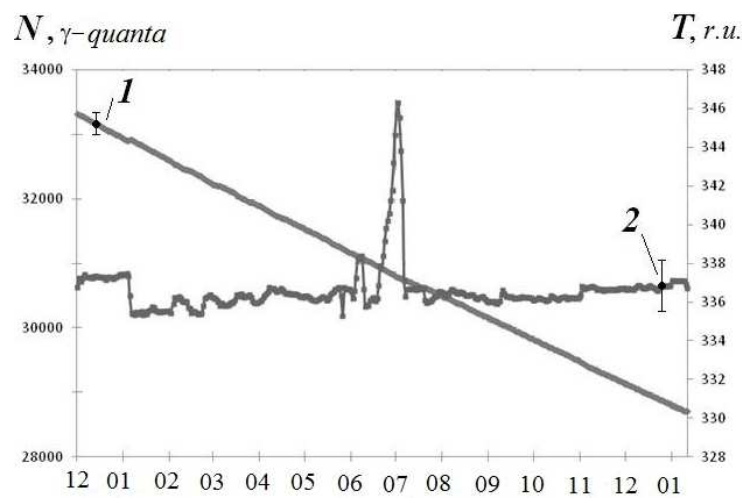

Fig. 9. Daily average $N$ - $\gamma$-quanta counts (1) with the threshold $>0.3 \mathrm{Mev}$ for the first channel and $T$ - daily mean temperature (2) in relative units (r.u.) for the stated period December 28, 2010 - February 08, 2012 (point on the abscises axis - the 28th of every month).

Two methods are used to analyze the results obtained: Fourier data sequence analysis method and Kolmogorov Smirnov (K-S) statistical method [5,14, 16, 17].

Let's repeat $\mathrm{K}-\mathrm{S}$ criterion is based on computation of maximum difference between the theoretical hypothesis of the uniform distribution of $\gamma$-quanta in the course of a day and the experimental distribution function. The criterion value for two decades under the experiment is shown in Fig. 4 and Fig. 5 on the vertical (K-S) axis. There is a table with $\mathrm{K}-\mathrm{S}$ tabulated values for the given hypothesis and obtained data correspondence probabilities. In the pictures the horizontal line with the significance level $(a), a=0.0125$ (Fig. 10) and $a=0.3$ (Fig. 11) corresponds to the criterion compliance probability. If $\mathrm{K}-\mathrm{S}$ goes above the horizontal line, the hypothesis is rejected with probability, for example, 0.9875 (Fig. 10), therefore there is statistically significant difference between the experimental and theoretical distributions. In the case under consideration, where theoretical distribution is selected to be uniform, this means non-uniformity of the measured value within the time of a day.

For the present study we selected the periods 10 days each. Intensity in the $\gamma-\gamma$ coincidence channel was under investigation. We were interested in a daily variation of $\gamma-\gamma$ counting. To increase accuracy within 24-hour period we added material 10 days in succession, i.e. the first point of the first day was matched with the first point of the second day, followed which the two rows were added. The same was made for the rest days. There is another one peculiarity of the present work. From the reference series only those intervals were selected (count for 10 seconds) in which the counting value exceeds the local average for $2 \sigma$, where $\sigma$ is a statistical error within the given interval. The series obtained in this way is to be considered below.
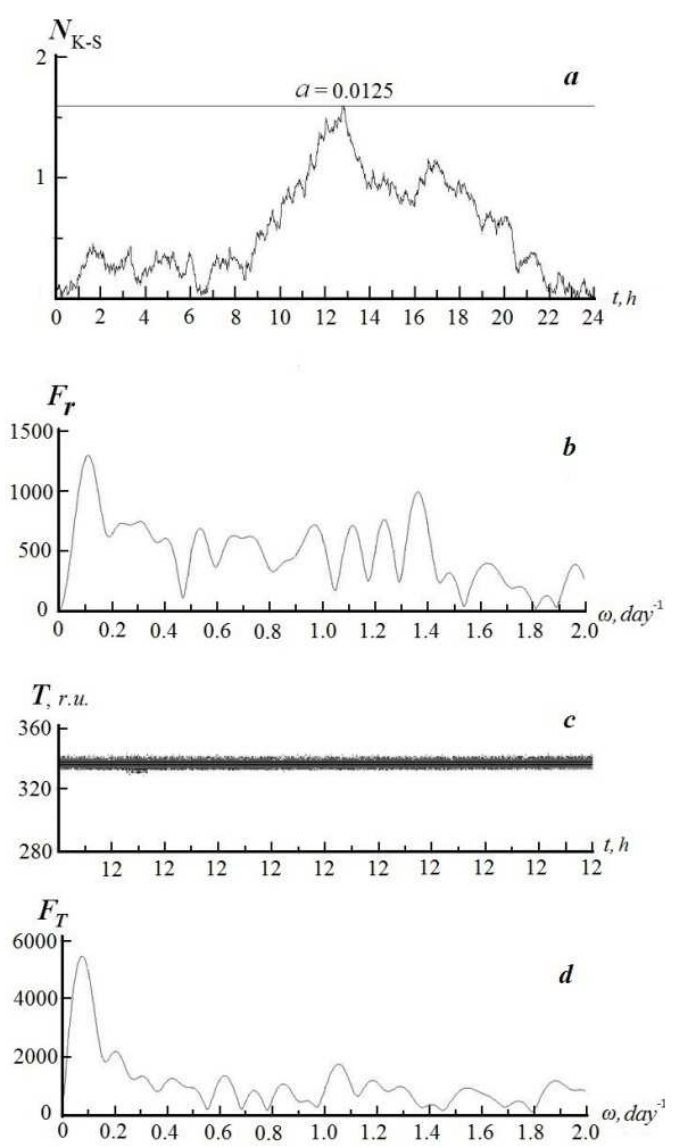

Fig. 10. Analysis results for the decade April, 22 - May, 02, 2011 in a coincidence series $(G / G)$ : a - non-uniformity assessment of $\gamma$-quanta series distribution, exceeding $2 \sigma$, by $K-S$ criterion ( $N_{K-S}$ criterion values are plotted against the axis of ordinates, the horizontal line corresponds to $N_{K-S}$ criterion value with the significance level $a=0.0125$ ) over the 24-hour period (the Moscow time $t$, hours, is plotted against the axis of abscissas); $b$ - Fourier spectrum $F_{R}$ for $\gamma$-quanta series, exceeding $2 \sigma ; c$ - the temperature series $T$ values in r.u.; $d$ - Fourier spectrum $F_{T}$ for the temperature series.

Estimation by $\mathrm{K}-\mathrm{S}$ test marked only four decades with the significance level $a$ less than 0.3:

March 11-21, with the significance level $a=0.1$; temperature correlation $\sim 0.05 \%$;

April 22 - May 02, with the significance level $a=0.0125$; temperature correlation $\sim-0.04 \%$; June 24 - July 04, with the significance level $a=0.05$; temperature correlation $\sim 2.1 \%$;

August 04 - 14, with the significance level $a=0.05$; temperature correlation $\sim 0.2 \%$.

As an example, Fig. 10 shows the analysis result for the decade April 22 - May 02, 2011. As it seen from the figure, the significance level of the result (non-uniformity of the decay over the 24-hour period) in its maximum reaches 0.0125 . Fourier analysis of the twenty-four hours harmonics also indicates the process non-uniformity. With that 
harmonics with the highest power for the main and temperature series do not coincide. Fig. 10 shows the temperature stability in relative units (r.u.) without hourly harmonics for the reference series (deviation $\pm 1.5 \%$ ). Figure 11 shows the analysis result for the decade July 23 - August 02, 2011, where the temperature perturbation was observed (Fig. 9) caused by heat wave in summer 2011 and thermostatic regulator failure to maintain preset temperature. As figure shows, the daily temperature harmonic, observable in the figure, practically does not influence the rise of the power of cophasal harmonic in the main $\gamma$-quanta spectrum. With that $\mathrm{K}-\mathrm{S}$ criterion value does not reach the limiting significance level of 0.3 .
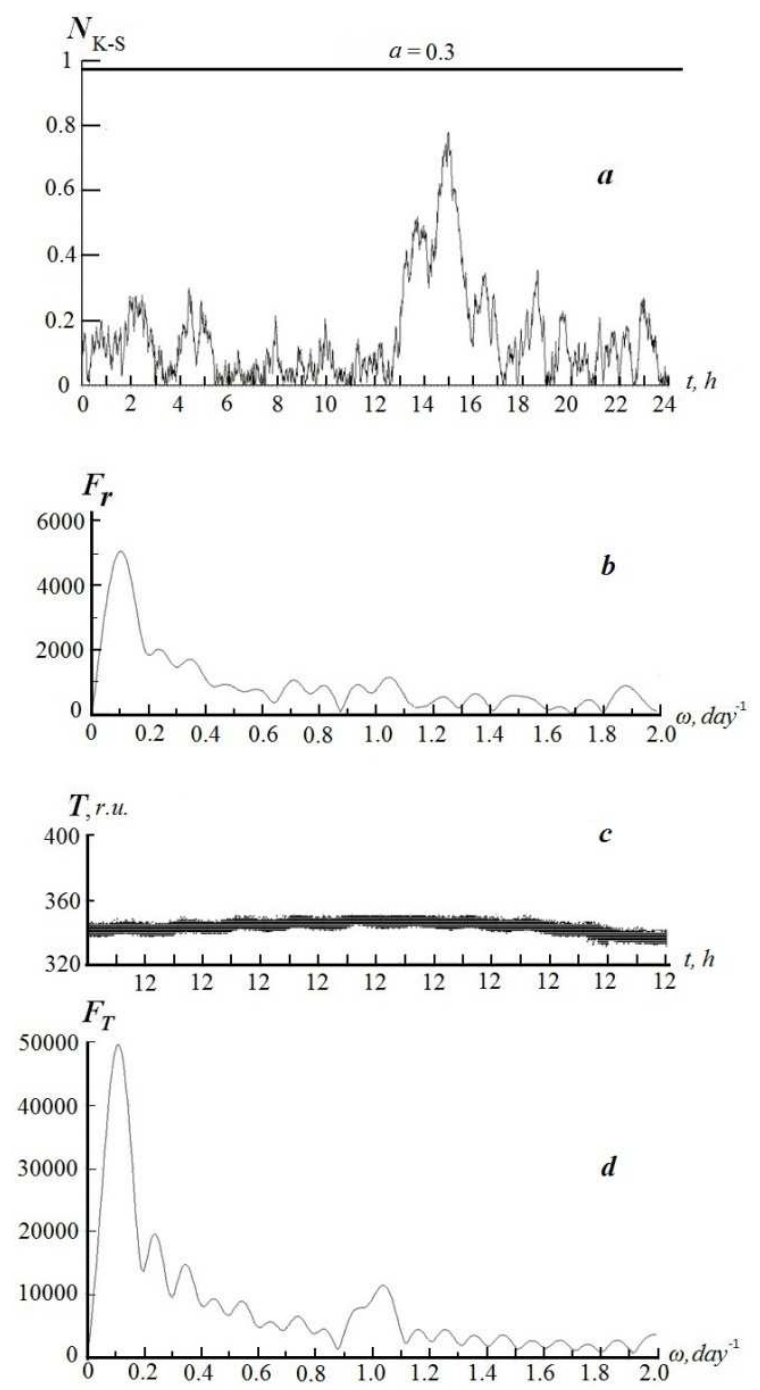

Fig. 11. Analysis results for the decade July 23 - August 02, 2011, in a coincidence series $(G / G)$ depending on $N_{K-S}$ criterion value (the horizontal line corresponds to $N_{K-S}$ value with the significance level $a=0.3$ ) over the 24hour period. All designations are the same as in the Figure 4.

Now consider possible long-periodic harmonics in ${ }^{60} \mathrm{Co}$ decay rate. A classical exponential decay with half life $T_{1 / 2}=$ $5.2 \pm 0.3$ years is observed, that corresponds to the tabular value 5.2713(8) years [36]. Measured values $T_{1 / 2}$, obtained by decades (selected decades with minimal temperature correlation) for the period December 28, 2010 - February 08, 2012, are shown in Figure 12. Some decades represent deviations from the tabular value $T_{1 / 2}$, but they are not beyond the measurement accuracy range.

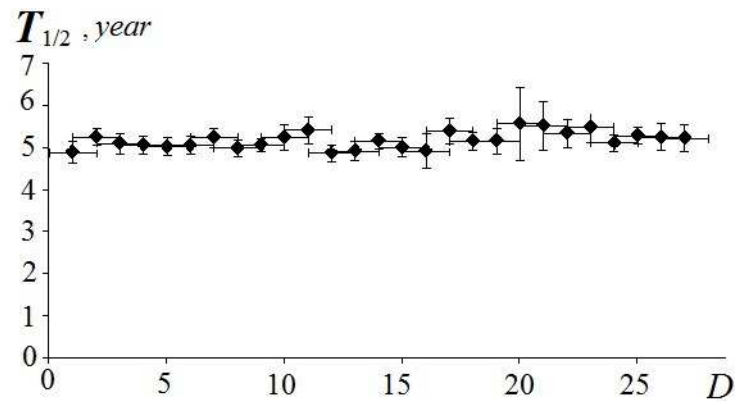

Fig. 12. The half-decay time $T_{1 / 2}$ of ${ }^{60}$ Co measured in 10 day intervals.

The period of time are selected points when temperature variation is small. $\mathrm{T}_{1 / 2}$ values are plotted against the axis of ordinates. Decades (D), the less temperature correlated, are plotted against the axis of abscissas. The standard deviations are shown.

10-days and 20-days periods in decay rate change were detected by applying Fourier analysis, which seems to be artefact caused by memory cards replacements. Additionally the data indicate the existence of 27-day period.

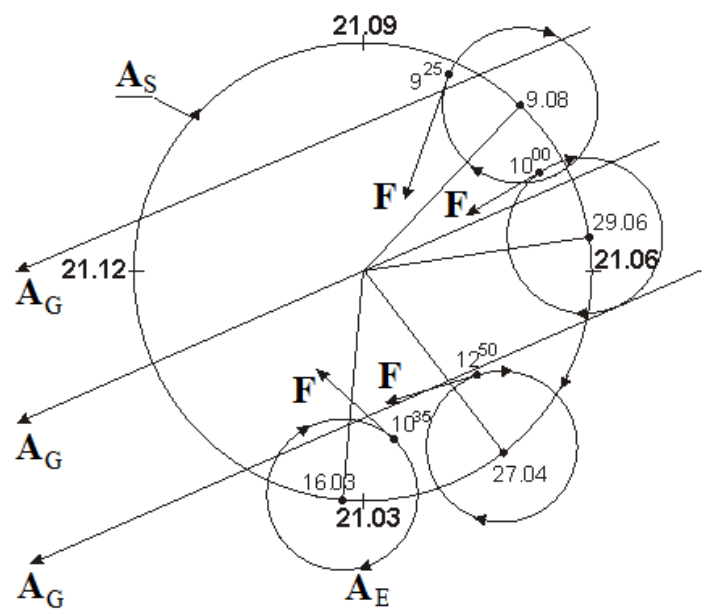

Fig. 13. The spatial positions of sites where the clearly expressed extrema in the magnitude of the flow of $\gamma$-quanta in the experiment with the $\beta$-decay of ${ }^{60} \mathrm{Co}$, were observed in [17] (see Figs 5,6 too).

$\leftarrow$ - the site of the maximum flow of $\gamma$-quanta with the indication of the direction of action of the new force $\mathrm{F}$ drawn along the tangent line to the parallel of latitude;

21.03 etc. - the point of the vernal equinox and other characteristic points of the trajectory of motion of the Earth and the radioactive source around the Sun;

$A_{E}$-the direction of the vectorial potential of the magnetic field of the Earth's dipole;

$A_{S}$-the direction of the vectorial potential of the magnetic field of the Sun's dipole;

$\mathrm{A}_{\mathrm{G}}-$ the direction of the cosmological vectorial potential.

The spatial positions of sites where the clearly expressed extremes in the magnitude of the flow of $\gamma$-quanta in the 
experiment with the $\beta$-decay of ${ }^{60} \mathrm{Co}$, were observed in [24, $25]$ are shown in Fig. 13. The direction of vector $A_{g}$ in Fig. 13 is the direction of the cosmological vectorial potential was defined in $[17,32,33]$ (right ascension $\alpha=293^{\circ} \pm 10^{\circ}$; declination $\delta=36^{\circ} \pm 10^{\circ}$.). We see that the directions of new force action in decades April 22 - May 02 and June 24 - July 04 coincides with the direction of vector $\mathrm{A}_{\mathrm{g}}$. The directions of new force action in decades March 11-21 and August 04 14 coincide with the generatrices of the revealed cone of new force action [17, 32, 33].

\section{Discussion and Conclusion}

Standard representations can't help in explaining the obtained last results, i.e. ${ }^{60} \mathrm{Co}$ decay rate changes and other results of this article connected with changes in $\beta$-decay rate of radioactive elements. Therefore it is taken to be a new force in nature that is able to influence neutron decay in ${ }^{60} \mathrm{Co}$ nucleus and the nucleuses other radioactive elements. The physics of assumed new force is described in beginning of article (see $[5,17]$ ), and its influence on the $\beta$-decay is discussed also in $[5,15,17,24,25]$. Let us briefly elucidate its nature. In the framework of a non-calibration theory of formation of physical space and world of elementary particles (Byuon theory), a part of the mass of elementary particle associated with the process of formation of its internal physical space is proportional to the modulus of summary potential $A_{\Sigma}$. The magnitude of $A_{\Sigma}$ modulus cannot be more than the modulus of the cosmological vectorial potential $\left|\mathbf{A}_{z}\right|$, a new fundamental constant got first in [1]. If the magnitude of $A_{\Sigma}$ is diminished by any way, the particle will be acted upon by the new force throwing out substance from the region with diminished modulus of $A_{\Sigma}$. In our experiments the magnitude of $\mathrm{A}_{\Sigma}$ decreases due to the action of the Earth's vectorial potential $A_{E}$ being in opposition to the vector $A_{g}$ practically at a tangent to the Earth's parallel. The cause is the decrease of the vector potential of the Sun's dipole component $A_{s}$ entering into $A_{E}$. In April 2011, As was directed like $A_{g}$, and October is in opposition to $A_{g}$. Therefore in April we can diminish the magnitudes of $\mathrm{A}_{\mathrm{s}}$ and hence $A_{g}$ due to $A_{E}$ but cannot in the second half year (from middle July till middle January) since in that time the vector $\mathrm{A}_{s}$ has no components directed equally with $\mathrm{A}_{\mathrm{g}}$ but only oppositely to $\mathrm{A}_{\mathrm{g}}$. It should be highlighted once more that in this case we cannot decrease masses of particles by the action of vector $A_{E}$. In this connection we see the maximum effect in April (for a given solar cycle).

Obtaining a result in one decade of August, 2011 (04.0814.08) and its absence in the same decade in 2010 is indicative of the approaching change in dipole component polarity of the Sun's magnetic field, which occurs every 11 years. The last similar process took place in 1999-2000 and lasted about 280 days [20]. After the Sun's magnetic dipole polarity is changed, the maximum effect is to be observed in October.

It is important to note that, for the time period being considered (from December 9, 1998, to April 30,1999), the anisotropy effect (period about $24 \mathrm{~h}$ ) became weaker and virtually disappeared from the middle of January 1999. It is clear because the maximum effect is to be observed in October.

In such a way the experiment results confirmed some previously obtained results. As well, they conform with anisotropic properties of the physical space, specified in [37], based on the annual oscillations data analysis while investigating ${ }^{32} \mathrm{Si}$ and ${ }^{226} \mathrm{Ra}$ half-decay periods $[10,11]$, being actively discussed in the references [19, 38, 39].

The quantity of works devoted by the investigation of decay rate changes of radioactive elements are ever increasing [40 - 42].

\section{Acknowledgments}

Authors express gratitude acad. RAS S.T. Belayev, Acad. RAS Yu.Z. Oganeseyan for support this investigations, Prof. V.A. Nikitin and other researchers from JINR (Dubna) because of participation in work [24, 25] and to all NUCLEUS-2011 International conference (October 10-14, 2011, Sarov, Russia), NUCLEUS-2012 International conference (June 25-30, 2012, Voronezh, Russia), NUCLEUS-2014 International conference (July 1-4, 2014, Minsk, Belarus) participants for fruitful discussion of the experimental results.

\section{References}

[1] Yu.A. Baurov, Yu.N.Babaev and V.K.Ablekov, Dokl. Akad. Nauk SSSR, vol. 259, No. 5, p. 1080, 1981.

[2] Yu.A. Baurov, Yu.N.Babaev and V.K.Ablekov, Dokl. Akad. Nauk SSSR, vol. 262, No. 1, p. 68, 1982.

[3] Yu.A. Baurov, Yu.N.Babaev and V.K.Ablekov,Dokl. Akad. Nauk SSSR (DAN), vol. 265, No. 5, p. 1108, 1982.

[4] Yu.N.Babaev, and Yu.A. Baurov, On the Origin of Fundamental Constants and Some Quantum Numbers, Preprint of Institute for Nuclear Research of the USSR Academy of Sciences, No. P-0362, 1984.

[5] Yu. A. Baurov "On the Structure of Physical Vacuum and a New Interaction in Nature (Theory, Experiment and Applications)", Nova Science, NewYork, 2000.

[6] Y.M.Shirokov, N.P.Yudin, Nuclear Physics, Second edition, Moscow, "Nauka", 1980 ,(in Russian).

[7] D. N. Trifonov, Radioactivity Yesterday, Today, Tomorrow,Atomizdat, Moscow, 1966, p. 91, (in Russian).

[8] T. Ohtsuki et al., Phys. Rev. Lett. 93, 112501 (2004).

[9] S. K. Godovikov, Pis'ma Zh. Erksp. Teor. Fiz. 79, 249; (2004) [JETP Lett. 79, 196 (2004)].

[10] D. E. Alburder, G. Harbottle, and E. F. Norton, Earth and Planet. Sci. Lett. 78, 169 (1986).

[11] H. Siegert, H. Schrader, and U. Schotzig, Appl. Radiat. Isot. 49, 1397 (1998).

[12] S. E. Shnol et al. Uspehi phys. Nauk, 168, 1129 (1998), (in Russian). 
[13] Yu. A. Baurov and V. L. Shutov, Prikladnaya Fizika, No. 1, 40 (1995)., (in Russian)

[14] Yu. A. Baurov, A. A. Konradov, V. F. Kushniruk and Yu. G. Sobolev, Scientific Report № E7-97-206, (Dubna, 1997), p.354.

[15] Yu. A. Baurov, A. A. Konradov, E. A. Kuznetsov et al., Mod. Phys. Lett. A 16, 2089 (2001).

[16] Y. A.Baurov, Yu.G.Sobolev, Y.B.Ryabov, V.F.Kushniruk., Phys. Atom. Nucl. 70, 1825 (2007).

[17] Yu. A. Baurov, Global Anisotropy of Physical Space. Experimental and Theoretical Basis (Nova Science, NY, 2004).

[18] Yu. A. Baurov, Bull. Russ. Acad. Sci. Phys. 76, 1076 (2012).

[19] J. C. Hardy, J. R. Goodwin and V. E. Iacob, Arxiv: 1108.5326.

[20] A. V. Derbin et al., Uspehi phys. Nauk, 170, 209 (2000) (in Russian).

[21] Yu.N.Babaev, and Yu.A. Baurov, Neutrino in discrete space and cosmology, Preprint of Institute for Nuclear Research of the USSR Academy of Sciences, No. P-0386, 1985.

[22] L.B.Okun', Leptons and quarks, Moscow, "Nauka", 1990 (in Russian).

[23] N.F.Nelippa, Physics of elementary particles, Moscow, "Vysshaya Shkola", 1977 (in Russian).

[24] Yu.A. Baurov, N. A. Demchuk, A. Yu. Baurov, A. Yu. Baurov (Jr..), V.B. Dunin, V.V. Tihomirov, S.V.Sergeev, Prikladnaya fizika 5, 12 (2011).

[25] Yu. A.Baurov, V.A. Nikitin, V. B. Dunin, N. A.Demchuk, A. Yu.Baurov, A. Yu.Baurov (Jr.), V. V. Tihomirov, S. V. Sergeev, arXiv:1304.6885 [nucl-ex].

[26] Yu.A. Baurov, Am. J. Mod. Phys., vol. 2, pp. 177-184, 2013.
[27] Yu.A.Baurov, E.Yu.Klimenko, and S.I.Novikov, DAN, v.315, p.1116, 1990.

[28] Yu.A.Baurov, E.Yu.Klimenko, S.I.Novikov, Phys. Lett. A., v.162 p.32, 1992.

[29] Yu.A.Baurov, P.M.Ryabov, DAN, v.326, p.73, 1992.

[30] Yu.A.Baurov, Phys.Lett. A., v.181, p.283, 1993.

[31] Yu. A. Baurov and A. V. Kopaev, Hadronic Journal, Vol. 25, pp 697-711, 2002.

[32] Yu.A. Baurov, et al., Phys. Lett. A311, p. 512, 2003.

[33] Yu.A. Baurov, A.G. Znak, V.G. Farafonof, Advances in Plasma Physics Reseach, Volume 5, pp. 179-196, editor Francois Gerard, 2007.

[34] Y.M.Shirokov, N.P.Yudin, Nuclear Physics, Second edition, Moscow, "Nauka", 1980 (in Russian).

[35] Yu.A.Baurov, A.A.Konradov, V.F.Kushniruk, Yu.G.Sobolev, in Scientific Report 1995-1996, "Heavy ion Physics", E7-97206, p.354-355, JINR, Dubna.

[36] G. Audi, O. Bersillon, J. Blachot and A. H. Wapstra, Nuclear Physics A 729, 3, 2003.

[37] Yu. A. Baurov, I. F. Malov, Int. J. Pure \& Appl. Phys. 6, 469. 2010.

[38] J. H. Jenkins et. al. Astro-ph/0808.3283, 25 Aug. (2008).

[39] 23. E. Fischbach et. al. Space Sci. Rev. 145, 285 (2009).

[40] Bellotti et.al. Phys. Lett. B,710, 114 (2012).

[41] J.H. Jenkins, E. Fischbach, D. JavorsekII, Robert H. Lee, P.A. Sturrock, Appl. Radiat. Isot. 74, 50 (2013).

[42] J. Nistora, J. Heima, E. Fischbach, J.H. Jenkins, P.A. Sturrock: arXiv:1407.4144 [nucl. ex]. 